\title{
Kernos
}

Revue internationale et pluridisciplinaire de religion

grecque antique

2| 1989

Varia

\section{Pierre Brulé, La fille d'Athènes. La religion des filles à Athènes à l'époque classique}

\section{Vinciane Pirenne-Delforge}

\section{(2) OpenEdition \\ Journals}

Édition électronique

URL : http://journals.openedition.org/kernos/259

DOI : 10.4000/kernos.259

ISSN : 2034-7871

Éditeur

Centre international d'étude de la religion grecque antique

Édition imprimée

Date de publication : 1 janvier 1989

Pagination : 260-262

ISSN : 0776-3824

\section{Référence électronique}

Vinciane Pirenne-Delforge, « Pierre Brulé, La fille d'Athènes. La religion des filles à Athènes à l'époque classique », Kernos [En ligne], 2 | 1989, mis en ligne le 04 mars 2011, consulté le 24 septembre 2020. URL : http://journals.openedition.org/kernos/259 ; DOI : https://doi.org/10.4000/kernos.259 
Pierre BRULÉ, La fille d'Athènes. La religion des filles à Athènes à l'époque classique. Mythes, cultes et société, Paris, Les Belles Lettres, 1987, 455 p., 35 fig., $24 \times 15,6 \mathrm{~cm}$ (Annales littéraires de l'Université de Besançon, 363. Centre de Recherches d'Histoire Ancienne, 76).

Se déclarant d'emblée l'héritier des travaux de Jeanmaire sur l'initiation, l'A. entend combler un vide laissé par ce genre d'étude en abordant la question de l'initiation féminine. Le contenu religieux de cette étape étant très marqué, il convient de dessiner un cadre général qui permette de comprendre la «religion» des filles. Le canevas de la recherche est donné par les vers 641-647 de la Lysistrata d'Aristophane qui parcourent quelques étapes des services religieux féminins à Athènes. Le plan de l'ouvrage en est déduit puisqu'il nous fait passer de l'arrhéphorie sur l'Acropole à l'arkteia de Brauron pour faire apparaître, dans sa troisième partie, l'image de la canéphore des Panathénées. L'A. aurait pu en rester là et nous livrer une interprétation des développements de ces trois gros chapitres. Il a préféré faire un détour par la démographie afin d'intégrer la fille dans un panorama global de société pour en terminer avec une brève comparaison entre filles et garçons en ce qui concerne leur intégration dans cette même société.

Le premier chapitre, intitulé "Athéna. En ville. L'Acropole féminine ou l'apprentissage du métier de femme», s'articule en trois parties principales : une étude minutieuse - presque microscopique - du mythe d'Érichthonios, la présentation des cultes et rituels acropolitains et, enfin, ce que l'A. appelle un "essai de mythe-fiction". Si Érichthonios se trouve au centre d'un complexe mythique, c'est qu'il incarne le "paradigme" du nourrisson athénien autour duquel vont s'affairer toute une galerie de personnages féminins qui serviront de référence aux jeunes athéniennes de l'histoire. Athéna, Aglauros/Agraulos, Pandrose, Hersè, Gê, Kourotrophos sont analysées successivement en fonction de leur(s) intervention(s) dans les différentes phases de développement d'Érichthonios : conception, naissance, «trophie». Avec l'étude des cultes et des rites, l'A. nous permet d'appréhender une initiation féminine aristocratique, qui tourne autour des tâches ménagères, sacralisées le temps d'un service religieux. Ainsi le broyage du grain (alétrie), le tissage du péplos d'Athéna (arrhéphorie), le nettoyage des vêtements et des statues de la déesse (plyntérie et kallyntérie). "Le travail apparaît comme une vertu... une dimension constitutive de l'existence quotidienne dans les oikoi, une forme de la permanence de la présence féminine auprès de la déesse; par le maintien immuable de ces composantes, il garantit la pérennité de la communauté» (p. 123). Mais l'arrhéphorie n'est pas que tissage. Pausanias (I, 27, 3) nous a conservé le souvenir d'un rituel nocturne qui fait intervenir les petites arrhéphores dans le transport d'objets mystérieux. Le texte du Périégète pose un problème d'interprétation qui concerne le but de ce voyage et l'A. nous livre un état de la question clair et 
précis. Les fillettes se rendent-elles dans le péribole d'Aphrodite év кńtorऽ ou dans un péribole «non loin de l'Aphrodite év kínoıs"? P. Brulé, qui interprète le rituel comme une "ordalie de virginité» - car la faute de la fille engage toute la cité - et qui parle très justement d'une initiation sexuelle "par allusion", commet, à notre avis, l'erreur de ne considérer la mention du sanctuaire d'Aphrodite que comme une référence "topographique» au sanctuaire de la déesse sur les bords de l'Ilissos. Alors qu'il est possible de rester sur l'Acropole, non loin du péribole d'Aphrodite et d'Éros découvert par Oscar Broneer! Même s'il ne s'agit pas du lieu d'arrivée des fillettes, il ne peut être question d'un tel déplacement de perspective. Et les comparaisons que l'A. entreprend avec des rites romains ne font que confirmer notre façon de voir.

La dernière partie de ce chapitre est très stimulante, même si l'A. reconnaît la hardiesse de son propos. Il émet, en effet, l'hypothèse qu'un mythe inconnu de nous aurait présenté Érichthonios comme un enfant exposé par une fille mère à la suite d'un viol, du type de la Créuse d'Euripide. Cette hypothèse s'intègre parfaitement dans l'ensemble de ce chapitre, mais ne perd rien, pour autant, de son caractère conjectural.

À l'âge où elles servent Athéna, certaines fillettes athéniennes sont susceptibles d'habiter hors de la ville, au service d'Artémis à Brauron (2e chapitre). Après une étude minutieuse du complexe mythique qui entoure les sites de Brauron, de Mounychie et d'Aulis, l'A. aborde les aspects topographiques des lieux de culte d'Artémis et d'Iphigénie, ainsi que leurs structures cultuelles communes (déplorons le manque de clarté de la fig. 25 p. 194) pour en revenir à l'analyse des éléments mythiques à grand renfort de tableaux et de schémas. Le thème des aitia est donc amplement développé avant que soit abordée l'étude du culte brauronien proprement dit. Les offrandes - qui concernent particulièrement le tissage et l'obstétrique - sont abondantes et révélatrices des attributions d'Artémis à Brauron, tandis que le rite de l'arkteia montre la fillette propitiant les divinités de la chasse et vivant «une initiation sexuelle qui est comme une mise à mort» (p. 260).

Avec le troisième chapitre, intitulé «Entre Artémis et Dionysos. La fille sur la place ou Porter-la-Corbeille», "le soleil canéphorique contraste violemment, avec la nuit des arrhéphores et des ourses. Si les secondes vivent une véritable initiation dans des sanctuaires fameux, les porteuses de corbeilles quittent leurs oikoi pour venir habiter un instant l'espace masculin à l'occasion d'un service religieux. Si les 'belles' filles se trouvent confrontées à l'amour' des hommes, les petites sont mises en présence d'animaux symboliques de la sexualité. Le passage de l'un à l'autre des systèmes se fait à la puberté» (p. 323).

Le quatrième chapitre tente de pallier la difficulté de parler des femmes dans un monde où seuls les hommes ont la parole. L'A. entend éviter l'écueil de la conception d'un féminin qui ne serait qu'un masculin «moins-quemasculin" et "mettre en évidence les éléments d'appréciation que fournit la 
société globale pour l'interprétation de la 'religion' des filles" (p. 341). Les épitaphes de femmes, les prescriptions sacerdotales et la littérature médicale sont successivement sollicitées afin de définir la fille aux différents âges de sa vie et la conclusion qui se dégage est fondamentale : dans le rapport au sacré, ce qui compte, c'est le rapport à l'homme, et c'est moins la virginité que la chasteté (p. 350), d'où l'importance des «vieilles» en cette matière. Et l'on peut se demander si le développement "démographique» que l'A. impose ensuite à son lecteur n'est pas une redondance ardue, d'autant plus inutile que ce genre d'étude dans le monde antique est inévitablement marqué au coin de l'incertitude.

La cinquième partie, plus courte, est consacrée à l'éventualité d'un rapport entre les initiations féminines et masculines, qu'il faut vraisemblablement rejeter.

Ce gros ouvrage est la publication de la thèse d'État de l'A. et il en présente les avantages et les inconvénients. On ne peut qu'applaudir devant une telle maîtrise d'un matériau aussi divers que dispersé, devant l'utilisation pertinente des sciences dites auxiliaires de l'histoire. On peut, sans trop se tromper, affirmer que l'A. a tout lu (la bibliographie est impressionnante), tout vu au cœur de son sujet et à la périphérie, ce qui fait de ce livre un outil de travail remarquable. Mais rien n'est épargné au lecteur qui suit pas à pas les cheminements du chercheur, et l'on se demande parfois si cette recherche n'aurait pas gagné à être résumée en maints endroits, sans pour autant rien perdre de sa richesse et de son intérêt. Mais, incontestablement, la fille d'Athènes s'anime et prend vie sous les yeux du lecteur de Pierre Brulé, dont le beau travail aurait mérité une impression de meilleure qualité.

Vinciane PIRENNE-DELFORGE

Métamorphoses du mythe en Grèce antique, sous la direction de Claude CALAME (voir les références complètes ainsi que le détail des articles cidessous à la rubrique «Actes de colloques»).

Claude Calame précise bien les données de l'étude actuelle du mythe dans son introduction - dont le titre évocateur juxtapose «l'évanescence du mythe» et «la réalité des formes narratives». Laissons-lui la parole en ce qui concerne les orientations du présent volume. "L'idée de l'«en soi» sémantique d'une réalité que l'on considère a priori comme constituant un mythe ou une mythologie est largement sous-jacente aux recherches présentées" (p. 7). "Avant de se poser la question d'une spécificité sémantique du mythe, la confrontation dont les recherches présentées ici sont le résultat visait à mettre en question les formes narratives et littéraires sous lesquelles nous est parvenu ce que nous croyons être la mythologie grecque» (p. 12). Le commentaire d'un volume de ce type est toujours un exercice périlleux. Les 